\title{
Effect of a transverse magnetic field on resonant magnetization tunneling in high-spin molecules
}

\author{
Jonathan R. Friedman and M. P. Sarachik \\ Department of Physics, The City College of New York, New York, New York 10031
}

J. M. Hernandez, X. X. Zhang, J. Tejada, and E. Molins

Facultat de Física, Universitat de Barcelona, 08028 Barcelona, Spain

R. Ziolo

Wilson Research Center, Xerox Corporation, Webster, New York 14580

\begin{abstract}
The recent observation of steps at regular intervals of magnetic field in the hysteresis loops of oriented crystals of the spin-10 molecular magnet $\mathrm{Mn}_{12} \mathrm{O}_{12}\left(\mathrm{CH}_{3} \mathrm{COO}\right)_{16}\left(\mathrm{H}_{2} \mathrm{O}\right)_{4}$ has been attributed to resonant tunneling between spin states. Here, we investigate the effect on the relaxation rate of applying the magnetic field at an angle with respect to the easy axis of magnetization. We find that the position of the resonances is independent of the transverse component of the field, and is determined solely by the longitudinal component. On the other hand, a transverse field significantly increases the relaxation rate, both on and off resonance. We discuss classical and quantummechanical interpretations of this effect. (C) 1997 American Institute of Physics.
\end{abstract}

[S0021-8979(97)53308-1]

$\mathrm{Mn}_{12}$ acetate $\left[\mathrm{Mn}_{12} \mathrm{O}_{12}\left(\mathrm{CH}_{3} \mathrm{COO}\right)_{16}\left(\mathrm{H}_{2} \mathrm{O}\right)\right]$, often referred to as $\mathrm{Mn}_{12}$, has been the subject of much recent research. ${ }^{1-5}$ First synthesized by Lis ${ }^{6}$ in 1980 , this molecular compound consists of weakly interacting ${ }^{4,5}$ magnetic cores of spin ground state $S=10$. A large negative magnetocrystalline anisotropy ${ }^{1}$ results in a barrier against spin reversal of $\sim 61-67 \mathrm{~K}$ (Refs. 2-5) and hysteretic behavior at low temperatures. ${ }^{2,5}$ Very recently, some of the present authors ${ }^{7}$ have obtained striking evidence for thermally assisted resonant tunneling in this material, a result now confirmed by others. ${ }^{8,9}$ The magnetic relaxation rate is found to increase markedly at regular intervals of magnetic field when the field is applied parallel to the easy axis of magnetization. This effect is attributed to resonant tunneling between energy levels in opposite wells of a double-well potential. In a simple model, ${ }^{7}$ illustrated in Fig. 1, pairs of levels become degenerate at regular intervals of field, and tunneling occurs between states near the top of the barrier.

In this paper, we present the results of a study of the effect on the magnetic relaxation of a symmetry-breaking transverse magnetic field. We find that for any value of the transverse field, the resonances always occur at the same values of longitudinal field. The application of a transverse field increases the relaxation rate both on and off resonance. The dependence on transverse field approximately follows the behavior one expects from the classical reduction of the energy barrier. There are subtle deviations from the classical form that may reflect the discrete energy level spectrum of this system.

Samples were prepared according to the published procedure. ${ }^{6}$ The data shown in Fig. 2 were obtained for a sample of oriented powder in an epoxy matrix. ${ }^{7}$ The data shown in Figs. 3 and 4 were taken on a single crystal of $\mathrm{Mn}_{12}$. Measurements of the dc magnetization were performed using a Quantum Design MPMS-5 magnetometer equipped with a 55-kOe superconducting magnet. The samples were mounted on a Quantum Design horizontal sample rotator, which allowed rotation of the easy axis of the sample with respect to the field in increments as small as $0.1^{\circ}$. Some systematic nonlinearity in the rotator can produce errors on the order of $1^{\circ}$ in the absolute angle. The desired values of the longitudinal and transverse field components were attained by adjusting the angle and the magnitude of the field. The component of the magnetization along the easy axis of the sample was inferred from the values of the components measured parallel and perpendicular to the field direction.

Figure 2(a) shows the magnetization parallel to the easy axis as a function of increasing longitudinal field for various fixed values of transverse field at $2.0 \mathrm{~K}$. The most striking feature is that the position of the steps is determined entirely by the longitudinal field component, and is clearly independent of the values of the transverse field. This is also clearly demonstrated by the derivative of the magnetization, shown in Fig. 2(b), where the peaks always occur at the same longitudinal-field values. On the other hand, the transverse field governs how the saturation magnetization is reached: the larger the transverse field, the smaller the longitudinal

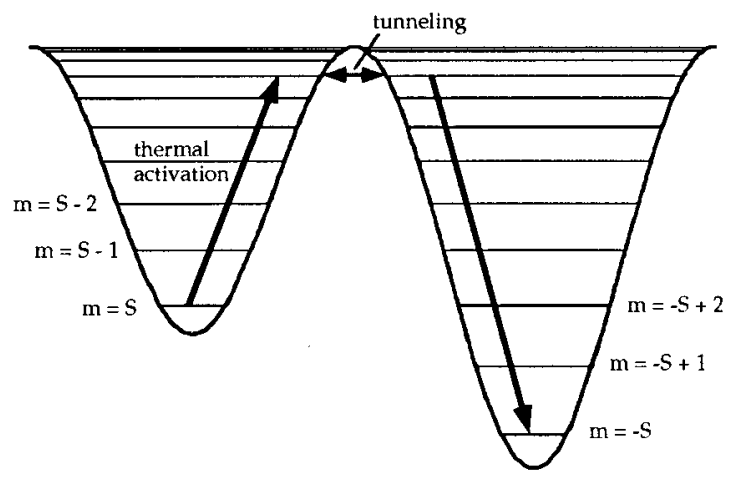

FIG. 1. Schematic diagram of the thermally assisted resonant tunneling process. Tunneling occurs when the field brings levels in opposite wells into resonance. The tunneling rate is fastest from levels near the top of the barrier. 


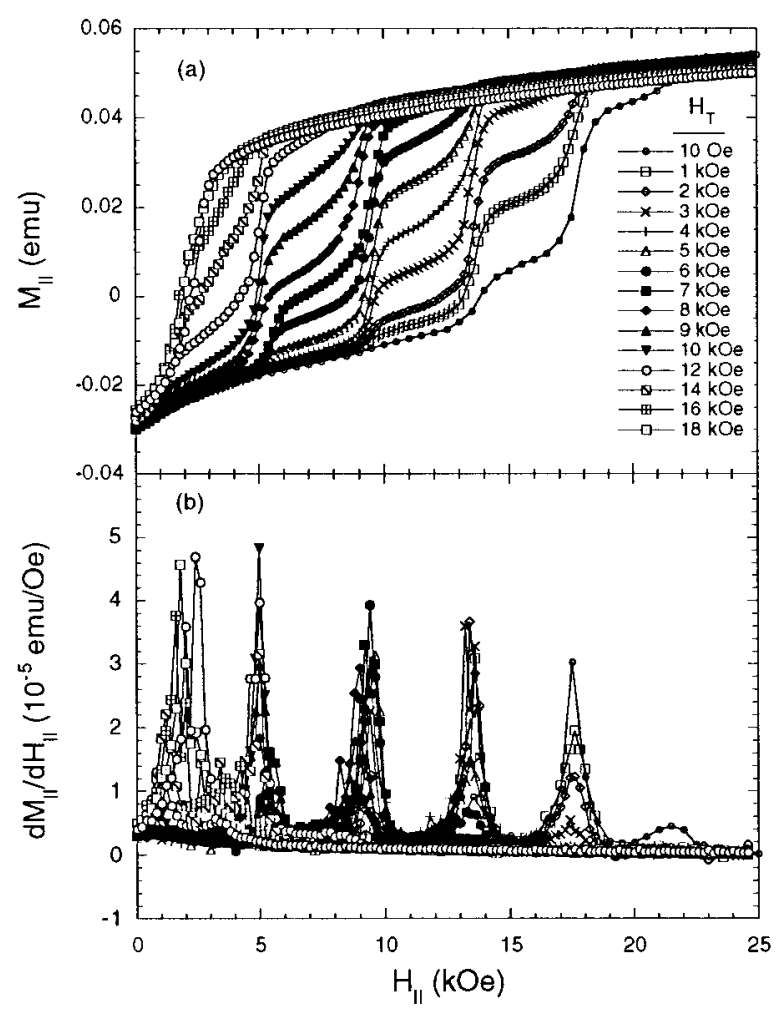

FIG. 2. (a) Magnetic moment as a function of external longitudinal field at $2.0 \mathrm{~K}$ for several values of transverse field. Note that this is one quarter of the hysteresis loops. The steps always occur at the same values of longitudinal field. (b) The derivative of the curves in (a).

field needed to reach saturation, i.e., the less hysteretic the behavior. Steps in the magnetization are observed in nearly all the curves. Note that some steps (at $H_{\|} \sim 9 \mathrm{kOe}$, for example) that are not apparent at small values of the transverse field become more pronounced as the transverse field is increased. This is consistent with the fact that the transverse field reduces the energy barrier, increasing the relaxation rate for all steps.

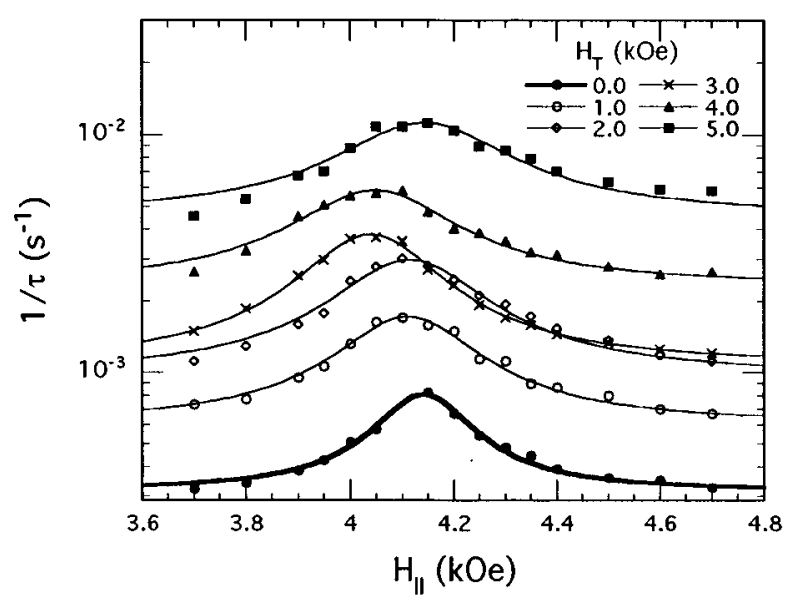

FIG. 3. The relaxation rate on a log scale as a function of the longitudinal field at $2.7 \mathrm{~K}$ for six values of transverse field, as indicated. The curves are fits to Lorentzian functions.

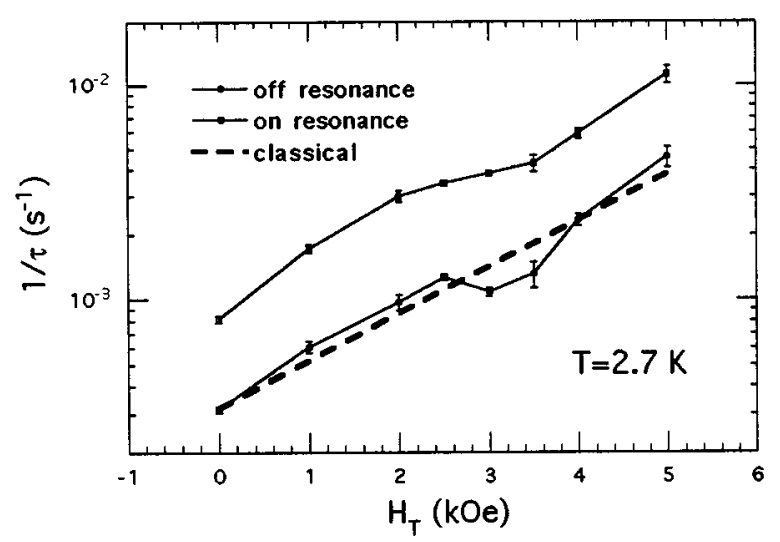

FIG. 4. The relaxation rate on resonance and off resonance (extracted from the fits in Fig. 3) as a function of transverse field. The dashed line is the expected classical result discussed.

Figure 3 shows the results of a detailed study of the resonance near $H_{\|}=4.1 \mathrm{kOe}$ as a function of both longitudinal and transverse field components. The sample was cooled in zero field to $2.7 \mathrm{~K}$, and a field was then applied at an angle to the sample's easy axis of magnetization. The relaxation rate was deduced by fitting the long-time tail of the relaxation curves to an exponential function. For consistency, the fit was applied to a subset of the data in which the moment is within $5 \times 10^{-5} \mathrm{emu}(\sim 0.1 \%$ of saturation) of its equilibrium value. The resulting relaxation rates were fitted to Lorentzian functions, shown by the curves in the figure.

The data reveal that the relaxation rate increases both on and off resonance as the transverse field is increased, consistent with a reduction in the barrier height. Also, the relaxation rate increases less sharply in a transverse field of $3 \mathrm{kOe}$ than for other values. There is a slight shift in the position of the peak as the transverse field is varied. This may be an experimental artifact and could be caused by an error in the angle of less than $1^{\circ}$. The width of the resonance also appears to roughly double from an initial value of $\sim 200$ Oe; this may also arise from slight errors in the angle.

One can extract relaxation times from the Lorentzian fits both on resonance (peak of the curve) and off resonance (background). The relaxation rates are plotted as a function of transverse field on a semilogarithmic scale in Fig. 4. Both on and off resonance, the relaxation rate appears to exhibit a plateau as the transverse field is increased. (The apparent nonmonotonic behavior in the off-resonance curve may not be significant, as it is based on a single point.) The onresonance and off-resonance curves have approximately the same average slope. One is simply shifted vertically relative to the other. The increase in relaxation rate expected from simple classical lowering of the energy barrier is denoted by the line labeled "classical" in Fig. 4. The slope of this line requires no adjustable parameters (the vertical intercept is, however, "pinned" to coincide with the zero-transversefield, off-resonant rate) and was obtained as follows. Classically, the relaxation rate is expected to increase with transverse field as

$$
\ln [\Gamma(H)] \propto-U_{0}\left(1-H / H_{c}\right)^{2} / k_{B} T
$$




$$
\approx-U_{0}\left(1-2 H / H_{c}\right) / k_{B} T \text { for } H \ll H_{C} .
$$

Here, $U_{0}$ is the magnitude of the classical energy barrier in zero field and $H_{c}=2 U_{0} / g \mu_{B} S$ is the field at which the barrier disappears. Substituting this expression for $H_{c}$, one finds that the relative change in the relaxation rate is $\ln [\Gamma(H) / \Gamma(0)] \approx\left(g \mu_{B} S / k_{B} T\right) H$. The dashed line in the figure was obtained from this expression using $g=2, S=10$ and $T=2.7 \mathrm{~K}$. The small, systematic oscillations of the relaxation rate about the classical result may arise from the discrete level structure of this spin-10 system, as discussed below.

The following Hamiltonian has been used to account for our earlier results:

$$
\mathscr{H}=-D S_{z}^{2}-g \mu_{B} \mathbf{S} \cdot \mathbf{B},
$$

where $D$ represents the magnetocrystalline anisotropy that breaks the zero-field Zeeman degeneracy. Within this model, illustrated in Fig. 1, the longitudinal and transverse field components play very different roles. When the field is purely longitudinal, the Hamiltonian is diagonal in $S_{z}$; the longitudinal field simply tilts the potential, making one well lower in energy than the other. A transverse field, on the other hand, breaks the symmetry of the Hamiltonian, splitting the degenerate pairs of states without tilting the potential. Friedman and Chudnovsky ${ }^{10}$ have calculated the effect of a transverse magnetic field on the values of longitudinal field, $B_{\|}$, for which the level crossings occur. Their results indicate that up to fourth order in perturbation theory the change in the resonance condition for $B_{\|}$is exactly zero for any value of $B_{\perp}$. This is borne out by the data shown in Fig. 2: As the transverse magnetic field changes, the values of the longitudinal field at which the resonances occur remain fixed.

The tunnel splitting of a large uniaxial spin in the presence of a transverse magnetic field has been calculated by Garanin ${ }^{11}$ in zero longitudinal field. The tunnel splitting, $E_{m}$, of two degenerate states with spin projections $\pm m$ is given by

$$
\Delta E_{m}=\frac{2 D}{[(2 m-1) !]^{2}} \frac{(S+m) !}{(S-m) !}\left(\frac{h}{2 D}\right)^{2 m},
$$

with $h=g \mu_{B} B_{\text {trans }}$. Using an estimated $0.01 \mathrm{~T}$ for the transverse field and the value of $D$ determined from our experiments, Eq. (2) yields the tunneling rates listed in Table I. The tunneling rates are very broadly distributed, with the rates of adjacent level crossings differing by several orders of magnitude. These results imply that tunneling from the ground states and first few excited states is negligible, while the tunneling between states near the top of the wells is so rapid that they are equivalent to a short. We suggest that tunneling is strongly dominated by one or two levels near the top of the well, and the time rate of decay is determined by the time required to repopulate these levels. Hence, on resonance the effective energy barrier is reduced: The system no longer needs to be thermally activated to the top of the barrier, but only to a fast-tunneling level at a somewhat lower energy. The decay rate may not be sensitive to the precise value of transverse field, being instead determined by the time required for thermal activation to the level that dominates the
TABLE I. Tunneling rates for different $m$ values calculated from Eq. (2), taken from Ref. 11, with $S=10$, and values $B_{\text {trans }}=0.01 \mathrm{~T}$ and $D=0.41$ $\mathrm{cm}^{-1}$.

\begin{tabular}{rc}
\hline \hline$m$ & Tunneling rate $(\mathrm{Hz})$ \\
\hline 1 & $3.2 \times 10^{8}$ \\
2 & $1.1 \times 10^{5}$ \\
3 & $3.5 \times 10^{0}$ \\
4 & $2.3 \times 10^{-5}$ \\
5 & $4.7 \times 10^{-11}$ \\
6 & $3.7 \times 10^{-17}$ \\
7 & $1.2 \times 10^{-23}$ \\
8 & $1.7 \times 10^{-30}$ \\
9 & $1.1 \times 10^{-37}$ \\
10 & $2.11 \times 10^{-45}$ \\
\hline \hline
\end{tabular}

tunneling. As one increases the transverse field, one expects abrupt changes in the relaxation rate at particular values of the transverse field, when the dominant tunneling process crosses over from one level to another. Numerical diagonalization of the Hamiltonian confirms the expectation that the energy barrier for a spin-10 system should exhibit sharp steps. ${ }^{12}$

The data of Fig. 4 show that the relaxation rate deviates subtly, but systematically, from the classical prediction. This behavior may indeed be evidence of the discrete level structure. However, it should be noted that a theoretical calculation predicts much clearer and sharper steps and plateaus than the subtle, "smeared out" behavior of Fig. 4. Measurements at a different temperature $(2.6 \mathrm{~K})$ nevertheless confirm the existence of the plateau. Further work is needed to determine the possible existence of such plateaus at other values of transverse field.

Acknowledgments: We are indebted to Eugene Chudnovsky for many useful and stimulating conversations. We thank Lucianne Walkowicz for her assistance in data processing. This research was supported by the U.S. Air Force Office of Scientific Research under grant No. F49620-92-J0190 .

${ }^{1}$ A. Caneschi, D. Gatteschi, R. Sessoli, A. L. Barra, L. C. Brunel, and M. Guillot, J. Am. Chem. Soc. 113, 5873 (1991).

${ }^{2}$ R. Sessoli, D. Gatteschi, A. Caneschi, and M. A. Novak, Nature (London) 365, 141 (1993).

${ }^{3}$ B. Barbara, W. Wernsdorfer, L. C. Sampaio, J. G. Park, C. Paulsen, M. A. Novak, R. Ferré, D. Mailly, R. Sessoli, A. Caneschi, K. Hasselbach, A. Benoit, and L. Thomas J. Magn. Magn. Mater. 140-144, 1825 (1995).

${ }^{4}$ M. A. Novak and R. Sessoli, in Quantum Tunneling of Magnetization edited by L. Gunther and B. Barbara (Kluwer, Dordrecht, 1995), pp. 171188.

${ }^{5}$ C. Paulsen and J.-G. Park, in Quantum Tunneling of Magnetization, edited by L. Gunther and B. Barbara (Kluwer, Dordrecht, 1995), pp. 189-207.

${ }^{6}$ T. Lis, Acta Crystallogr. Sec. B 36, 2042 (1980).

${ }^{7}$ J. R. Friedman, M. P. Sarachik, J. Tejada, J. Maciejewski, and R. Ziolo, J. Appl. Phys. 79, 6031 (1996); J. R. Friedman, M. P. Sarachik, J. Tejada, and R. Ziolo, Phys. Rev. Lett. 76, 3830 (1996); J. M. Hernandez, X. X. Zhang, F. Luis, J. Tejada, J. R. Friedman, M. P. Sarachik, and R. Ziolo, (to be published in Phys. Rev. B ).

${ }^{8}$ J. M. Hernandez, X. X. Zhang, F. Luis, J. Bartolome, J. Tejada, and R. Ziolo, Europhys. Lett. 35, 301 (1996).

${ }^{9}$ L. Thomas, F. Lionti, R. Ballou, D. Gatteschi, R. Sessoli, and B. Barbara, Nature (London) 383, 145 (1996).

${ }^{10}$ J. R. Friedman and E. M. Chudnovsky (unpublished, 1996).

${ }^{11}$ D. A. Garanin, J. Phys. A 24, L61 (1991).

${ }^{12}$ J. R. Friedman, Ph.D. thesis, The City College of CUNY, September 1996. 